\title{
Tunnel Diode Modeling, Including Nonlocal Trap-Assisted Tunneling: A Focus on III-V Multijunction Solar Cell Simulation
}

\author{
Mathieu Baudrit and Carlos Algora. Senior Member, IEEE
}

\begin{abstract}
Multijunction solar cells (MJCs) based on III-V semiconductors constitute the state-of-the-art approach for highefficiency solar energy conversion. These devices, consisting of a stack of various solar cells, are interconnected by tunnel diodes. Reliable simulations of the tunnel diode behavior are still a challenge for solar cell applications. In this paper, a complete description of the model implemented in Silvaco ATLAS is shown, demonstrating the importance of local and nonlocal trap-assisted tunneling. We also explain how the measured doping profile and the metalization-induced series resistance influence the behavior of the tunnel diodes. Finally, we detail the different components of the series resistance and show that this can help extract the experimental voltage drop experienced by an MJC due to the tunnel junction. The value of this intrinsic voltage is important for achieving high efficiencies at concentrations near 1000 suns.
\end{abstract}

Index Terms-III-V semiconductors, simulation, solar cell, tunnel diode.

\section{INTRODUCTION}

$\mathbf{T}$ UNNELING through a highly doped p-1l junction is a phenomenon tightly related to quantum theory. Quantum theory allows that a carrier with low energy has a finite probability of jumping to the other side of an energy barrier without increasing its energy. The carrier does not go over the barrier, which would require energy absorption; rather, it passes through the energy barrier (tunnels) and retains its original energy. This is a phenomenon with many applications in electronic devices [1]. Solar cell research has gained increasing attention in receut years. Iu monolithically grown multijunctiou solar cells (MICs), the individual subcells are interconnected via tunnel diodes. They must have both a low series resistance to miuimize the voltage drop betweeu subcells aud a high optical trausmissivity to minimize the curreut losses resultiug from light absorption inside the tunnel junction layers.

Since the $1960 \mathrm{~s}$, when solar cell concepts were first developed in earuest, new structural and material research has emerged, leading toward higher efficiency devices. Among high-efficiency concepts, MJCs have overcome the $40 \%$ efficiency barrier, setting records of up to $41.1 \%$ [2] at 454 suns using triple-junction devices and achieving yields of $32.6 \%$ efficiency at 1000 suns using dual-junction devices [3]. When deployed with solar concentrator systems, these devices have become true contenders in the marketplace. The high efficiencies achieved by these devices arise from complexities that make experimental optimization complicated, time consuming, and expensive. Numerical modeling, therefore, is a valuable tool for automating solar cell design and optimization, decreasing costs and development time. Some reliable modeling results have been already obtained for III-V single-junction solar cells using commercially available semiconductor simulation tools [4]-[6]. To do the same for MICs, a suitable model for the tunnel diode is necessary.

Efforts have been made to model tunnel junctions using classic models developed by Hurkx et al. [7], Klaassen, or Kane [8] without success [9]. These models are local models and have been developed for tunneling processes at gate interfaces in MOS transistors. The assumptions may not be applicable to tunnel diodes within MJCs. Tunneling is nonlocal in MJCs, as demonstrated in [10], in contrast to local tunneling at gate interfaces in MOS transistors.

Development of an appropriate tunnel diode model has not yet been achieved for use in simulations of MJCs. Reliable and realistic simulations can ouly be obtaiued by integrating accurate models for the tuuuel diode behavior iuside solar cells [10]. This kind of study has been already realized in [10] with satisfactory results; however, some mismatches appear in the valley regiou of the tuunel diode $J-V$ curve as the model does not include noulocal trap-assisted tuuueling (TAT). Therefore, iu this paper, we aualyze the model implemeuted iu Silvaco ATLAS [11] and describe its behavior when modeling tunnel diodes alone, as well as inserted into more complex structures. Particular emphasis is placed ou the local aud nonlocal TAT effects to show that these models are fundaneutal to reproduce the behavior of tunnel diodes over the whole voltage range, as it can be observed in [12], where adequate optimization methods, including the tunnel junction model presented inhere, revealed uew paths for MJC efficieucy improvement. The tunueliug models are here verified by comparing experimental measuremeuts with simulatious, accoutiug for the real doping profile. Finally, we focus on the tunnel diode behavior inside a solar cell. We describe how the models can help extract the series resistauce effects due to the tunuel junctiou from the series resistauce effects inhereut to the semicouductor structure. 


\section{TUNNELING EFFECT}

\section{A. Model Description}

A p-11 junction is usnally built nsing lightly doped materials with a low concentration of impnrities; notwithstanding, in 1958, the Japanese scientist Leo Esaki created a p-n junction using highly doped semiconductors with an impurity concentration of $1 \times 10^{19} \mathrm{~cm}^{-3}$, which caused them to degenerate. The new junctions showed a region of differential negative resistance that had not been seen before in any other device [13]. When a sufficiently high electric field exists within a p-11 junction, local bands inay bend sufficiently to allow electrons to tunnel by internal field emission from the valence band into the conduction band; the symmetric behavior occurs for holes. An additional electron, therefore, transfers to the conduction band, and a hole transfers to the valence band. Local band-to-band tunneling models can be employed to reproduce the behavior of such devices. These models, when implemented inside a technology computer-aided design (TCAD) environment, use the electric field value at each node ${ }^{l}$ along the junction to give a generation rate at that point due to tunneling. In reality, the tunneling process is nonlocal, and it is necessary to account for the spatial profile of the energy bands. This is why local models, such as those presented by Hurkx, Klaassen, or Kane, do not work properly and cannot correctly reproduce the characteristics of these devices near a zero voltage bias, as explained in [10]. It is also necessary to account for the spatial separation of the electrons generated in the conduction band from the holes generated in the valence band. A model for this process has been created for Silvaco ATLAS software. The model assumes that tunneling is 1-D in nature, so that it can be calculated using a special rectangular mesh of nanometer size superimposed over and coupled to the ATLAS regular mesh. At moderate doping levels, a tunneling effect can be seen under reversebiased conditions, but if the junction doping levels are high enough, the tunneling effect can also appear under forwardbiased conditions. If only elastic scattering mechanisms are considered, electrons from anywhere in the permitted energy range, constituted by the depletion region, can tunnel from the valence band to the conduction band. ATLAS considers each energy in the allowed range and determines the beginning and ending spatial positions, i.e., $x_{\text {reg }}$ and $x_{\text {end }}$, respectively, for tunneling at each energy $E$. The contribution to the tunneling current, for an electron in the energy range from $E-\Delta E / 2$ to $E+\Delta E / 2$ (where $\Delta E$ is a sinall energy increment) is therefore [11]

$$
\begin{array}{r}
J(E)=\frac{q k T}{2 \pi^{2} \hbar^{3}} \tau(E)\left(m_{e}^{*} \ln \left\{\frac{1+\exp \left[\left(E-E_{\mathrm{FI}}^{e}\right) / k T\right]}{1+\exp \left[\left(E-E_{\mathrm{Fr}}^{e}\right) / k T\right]}\right\}\right. \\
\left.-m_{h}^{*} \ln \left\{\frac{1+\exp \left[\left(E-E_{\mathrm{FI}}^{h}\right) / k T\right]}{1+\exp \left[\left(E-E_{\mathrm{Fr}}^{h}\right) / k T\right]}\right\}\right) \Delta E
\end{array}
$$

where $\tau(E)$ is the tunneling probability; $T$ is the temperature; $k$ is the Boltzinann's constant; $q$ is the electron charge; $\hbar$ is the

\footnotetext{
When simulating in a TCAD enviromment, the device is separated into indivisible elements, squares or triangles. to constitute a mesh. A node is a point at which more than two of these elements are in conlact.
}

reduced Planck's constant; $E_{\mathrm{FI}}$ and $E_{\mathrm{Fr}}$ are the Fermi levels on the left and right sides of the tnnnel junction, respectively, for holes or electrons (indicated by the superindex); and

$$
\begin{aligned}
& m_{e}^{*}=m_{i)} \sqrt{m_{e}\left(x_{\text {end }}\right) m_{h}\left(x_{\text {beg }}\right)} \\
& m_{h}^{*}=m_{i)} \sqrt{m_{e}\left(x_{\text {beg }}\right) m_{h}\left(x_{\text {end }}\right)}
\end{aligned}
$$

where $m_{e}$ and $m_{h}$ are the effective masses for electrons and holes, respectively, and $m_{0}$ is the free-electron rest mass. The contribution to the tunneling current is calculated and coupled into the mesh at $x_{\text {beg }}$ and $x_{\text {end. This is repeated }}$ over the whole range of allowed tunneling energies. Therefore, the tunneling model modifies the continuity equations with generation terms (reverse bias) or recombination terns (forward bias). The tunneling probability is calculated using a Wentzel-Kramers-Brillouin (WKB) two-band approximation [14]-[16] for the evanescent wave vector

$$
k_{v}=\frac{k_{e} k_{h}}{\sqrt{k_{e}^{2}+k_{h}^{2}}}
$$

where

$$
\begin{aligned}
& k_{e}=\frac{1}{i \hbar} \sqrt{2 m_{0} m_{e}\left(E-E_{c}\right)} \\
& k_{h}=\frac{1}{i \hbar} \sqrt{2 m_{0} m_{h}\left(E_{v}-E\right)}
\end{aligned}
$$

and $E_{c}$ and $E_{v}$ are the conduction and valence band energies, respectively.

This formulation ensures that the tunneling is electron like near the conduction band, hole like near the valence band, and mixed at energies near the middle of the energy gap. Direct quantum tunneling simulations often use a transmission matrix method to calculate the tunneling probability; however, in the case of band-to-band tunneling, a carefully applied WKB method, such as the one shown in this paper, is found to give equivalent results when used to calculate $\tau(E)$ and is computationally more efficient [11].

\section{B. Trap Effects in Tinnel Diodes}

During the growh process of semiconductor devices, it is not rare to involuntarily create a deep level inside the band gap. Such levels, due to defects and traps, influence the generation-recombination mechanisms. Although these effects are generally not dominant in regular p-11 junctions, in tunnel diodes, they can strongly modify the characteristics of the device. At high electric fields, local TAT is dominant and can affect the peak current value. As the electric field decreases, nonlocal TAT increasingly modifies the negative resistance and valley regions of the tunnel diode.

To reliably simulate tunnel diodes, it is important to incorporate a complete model for both local and nonlocal TAT, particularly when the model is intended to be used in MJC simulations. Indeed, MJCs are used under an optical concentrator, which implies a nonuniform distribution of light over the device surface. In this case, the light distribution can lead 


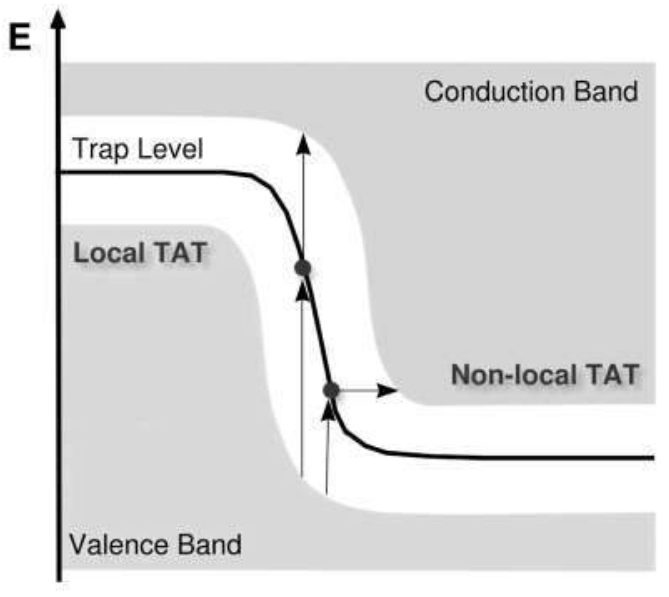

Fig. 1. Band diagram explaining local and nonlocal TAT.

to localized currents that are higher than the peak current supported by the tunnel diodes. This situation highlights the importance of reproducing the behavior of tunnel diodes over the whole voltage range, including the valley region, because local parts of these devices may potentially work in this regime.

1) Local TAT: TAT models describe the trap-to-band phonon-assisted tunneling effects resulting from deep-level traps inside the band gap, as shown in Fig. 1. At high electric fields, the tunneling of electrons from the valence band to the conduction band through trap or defect states can have an important effect on the current. TAT is modeled by including appropriate enhancement factors $\left(\Gamma_{n}^{\mathrm{DIRAC}}\right.$ and $\Gamma_{p}^{\mathrm{DIRAC}}$ ) in the trap lifetimes given in (7) and (8). These enhancement factors modify the lifetimes so that they include the effects of phononassisted tunneling on the emission of electrons and holes from a trap.

For donor-like traps, the recombination term for traps becomes (7), shown at the bottom of the page, where $\tau_{n}$ is the electron lifetime; $g_{D}$ is the degeneracy factor; $T_{L}$ is the lattice temperature; $n_{\mathrm{ie}}$ is the intrinsic carrier concentration; $n$ and $p$ are the electron and hole concentrations, respectively; and $E_{t}$ is the trap energy level. The recombination term for acceptorlike traps becomes (8), shown at the bottom of the page, The field-effect enhancement factor for electrons is given by

$$
\Gamma_{n}^{\text {DIRAC }}=\frac{\Delta E_{n}}{k T} \int_{0}^{1} \exp \left(\frac{\Delta E_{n}}{k T_{L}} u-K_{n} u^{3 / 2}\right) d u
$$

and the field-effect enhancement factor for holes is given by

$$
\Gamma_{p}^{\text {DIRAC }}=\frac{\Delta E_{p}}{k T} \int_{0}^{1} \exp \left(\frac{\Delta E_{p}}{k T_{L}} u-K_{p} u^{3 / 2}\right) d u
$$

where $u$ is the integration variable, $\Delta E_{n}$ is the energy range in which tunneling can occur for electrons, $\Delta E_{p}$ is the tunneling energy range for holes, and $K_{n}$ and $K_{p}$ are defined as

$$
\begin{aligned}
K_{n} & =\frac{4}{3} \frac{\sqrt{2 m_{0} m_{\text {traps }} \Delta E_{n}^{3}}}{3 q \hbar|E|} \\
K_{p} & =\frac{4}{3} \frac{\sqrt{2 m_{0} m_{\text {traps }} \Delta E_{p}^{3}}}{3 q \hbar|E|}
\end{aligned}
$$

where $|E|$ is the absolute value of the electric field, and $m_{\text {traps }}$ is the effective mass used in local TAT. Local TAT can be tuned to reproduce the behavior of specific traps by specifying $m_{\text {traps }}$.

The approximations made to evaluate the field enhancement factors $\Gamma_{n}^{\text {DIRAC }}$ and $\Gamma_{p}^{\text {DIRAC }}$ for local TAT result in an inability to account for the nonlocal TAT effects. However, a more accurate version of this model, i.e., the nonlocal TAT model, can reproduce both local and nonlocal TAT.

2) Nonlocal TAT: The TAT model explained above uses approximated field-effect enhancement factors. They are evaluated using an estimate for the tunneling probability. It is assumed that the tunneling probability at each point depends only on the local field strength and that the field is constant over the spatial range in which tunneling is allowed. This gives Airy function solutions for the tunneling probabilities. A further approximation is also made by replacing the Airy function with the leading term of its high-field asymptotic behavior. The integration over allowed tunneling energies is also approximated. A more accurate expression for the fieldeffect enhancement factor is

$$
\Gamma_{n}^{\text {DIRAC }}=\frac{1}{k T} \int_{0}^{\Delta E_{n}} e^{E / k T} \tau_{\text {traps }}(E) d E
$$

where $\tau_{\text {traps }}(E)$ is the probability that an electron with energy $E$ elastically tunnels into the conduction band from the trap position. The exponential term gives the probability that the electron will gain energy from phonon interactions before tunneling. In (13), the energy reference is the conduction band edge and is the maximum energy for which tunneling is

$$
R_{D}=\frac{p n-n_{\mathrm{ie}}^{2}}{\frac{\tau_{n}}{1+\Gamma_{n}^{\text {DIRAC }}}\left[p+g_{D} n_{\mathrm{ie}} \exp \left(\frac{E_{i}-E_{t}}{k T_{L}}\right)\right]+\frac{\tau_{p}}{1+\Gamma_{p}^{D I R A C}}\left[n+g_{D} n_{\mathrm{ie}} \exp \left(\frac{E_{t}-E_{i}}{k T_{L}}\right)\right]}
$$

$$
R_{A}=\frac{p n-n_{\mathrm{ie}}^{2}}{\frac{\tau_{n}}{1+\Gamma_{n}^{\text {DIRAC }}}\left[p+g_{A} n_{\mathrm{ie}} \exp \left(\frac{E_{i}-E_{t}}{k T}\right)\right]+\frac{\tau_{p}}{1+\Gamma_{p}^{D I R A C}}\left[n+g_{A} n_{\mathrm{ie}} \exp \left(\frac{E_{t}-E_{i}}{k T}\right)\right]}
$$




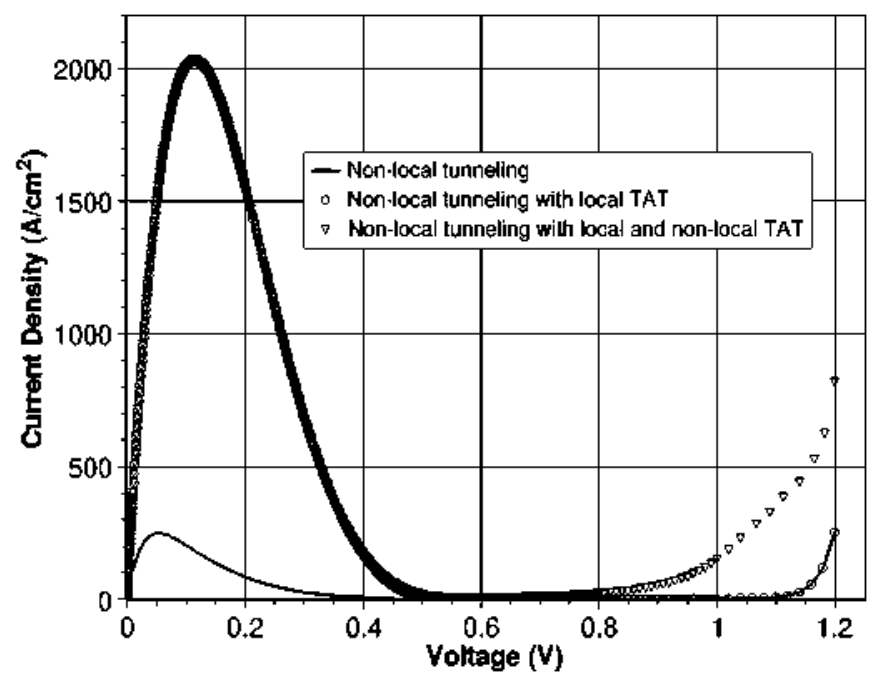

Fig. 2. Simulated tunnel diode $J-V$ curves with the (solid line) nonlocal tunneling model, (circles) nonlocal tunneling model with local TAT, and (triangles) nonlocal tunneling model with nonlocal TAT.

possible; or it is the trap level, if its value is lower than this maximum. There is a similar expression for holes. This precise model is able to handle the TAT effects when they locally occur (local TAT), without spatial carrier transport, and nonlocally (nonlocal TAT), with spatial carrier transport.

The tunneling probability $\tau_{\text {traps }}(E)$ is evaluated using a WKB method. The integral in (13) is numerically evaluated to give the terms $\Gamma_{n}^{\text {DIRAC }}$ and $\Gamma_{p}^{\text {DIRAC }}$. These terms are then used in (7) and (8). The values for the wave vectors used in the tunneling probability calculation are evaluated using the density-of-states effective mass. It should be noted that the effective mass is assumed to be constant throughout the tunneling process.

\section{TUNNEL DiOdE SimUlation}

\section{A. Semiconductor Stricture and Model Infuence}

To test the models presented above, simulations must be carried out. The first step is to identify the best tunnel diode model that allows agreement between simulation and experimental data. Indeed, a real tunnel diode consists of more than just a highly doped p-1l junction [17]. As previously shown, when simulating GaInP solar cells [5], the layers surrounding the active layers can modify the results. The importance of this effect should be determined, along with identification of the dominant effects included in the model, to properly reproduce the behavior of a tunnel diode.

1) Model Influence: The first simulation nu was carried out using a GaAs p $\mathrm{p}^{++}\left(5 \times 10^{19} \mathrm{~cm}^{-3}\right) / \mathrm{n}^{++}\left(3 \times 10^{19} \mathrm{~cm}^{-3}\right)$ tunnel diode to characterize the $J-V$ curves under each model. All model parameters were set to the default values; the goal of these simulations was not to adjust the parameters and models but to provide evidence for the models' ranges of influence.

Fig. 2 represents the simulated tumnel diode $J-V$ curve with the nonlocal tunneling model, the nonlocal tunneling model coupled with local TAT, and the nonlocal tinneling model coupled with nonlocal TAT.
The results show that inclnding TAT mechanisms in the tnnnel diodes simulation strongly inflnences the peak current $J_{p}$, increasing it from 250 to $2080 \mathrm{~A} / \mathrm{cm}^{2}$. This augmentation also leads to a major peak voltage $V_{p}$ that shifts from 0.05 to $0.12 \mathrm{~V}$. Calculating the series resistance of these devices, the value of which decreases from 0.2 to $0.06 \mathrm{m \Omega} \cdot \mathrm{cm}^{2}$, we can deduce that the changes in $V_{p}$ are due not only to an increased peak current value but also to TAT. Generally, traps are not desired in solar cells because they decrease the global efficiency by reducing the minority carrier lifetime and shunt resistance. However, a tunnel diode must act as a resistance with a very low resistivity. Therefore, traps become tumeling catalysts. Because local and nonlocal TAT inodels only differ in the calculation of $\Gamma^{\text {DIRAC }}$, a comparison of the two simulation results shows that the local model's approximation is correct at high electric fields. The results show identical behavior in the first part of the $J-V$ curve for the tunnel diode studied. In spite of this, the TAT models diverge at voltages above $0.8 \mathrm{~V}$. Because of the model's approximations, the local TAT model does not consider tunneling at low electric fields and results in behavior that is identical to the simple nonlocal tunneling model without TAT. The nonlocal TAT model shows a very different behavior in the valley region. As mentioned, a nomuniform light distribution on the MJC during operation inside an optical concentrator can lead to localized currents that are higher than the peak current supported by the tunnel diodes. It is therefore important to reproduce the behavior of tunnel diodes over the whole voltage range, including the valley region, because local parts of these devices may potentially work in these regimes. These simulations suggest some clues to data fitting, particularly concerning the presence of traps. As shown in Fig. 2, traps can assist the tunneling effect by both local and nonlocal TAT and strongly modify the behavior of the tunnel diode.

2) Semiconductor Structure Infinence: After analyzing the models' effects on tunnel diode behavior, we investigated the influence of the simulated device structure on the results. Tunnel diodes usually have barrier layers to prevent dopant diftusion [18], [19], cap layers, and a subsirate that acts as a mechanical support [17]. The central processing unit time required to obtain a solution is proportional to $N^{\alpha}$, where $N$ is the discretization used, and $\alpha$ varies from 2 to 3 , depending on the complexity of the structure. Thus, the most time-efficient way to model a device is to simulate the regions of interest, allocating a fine discretization only in critical areas and a coarse discretization elsewhere. It is important first to quantify the effect of the layers that do not correspond to the active regions [5]. To address this point, three tunnel diodes were modeled: one included only the active layers; the second included the whole structure; and a third included the real doping profile of the active region inside the structure. The simulations were carried out with the nonlocal tunneling effect and local TAT. The goal was to principally focus on the first part of the $J-V$ curve, the normal working region of the tunnel diode in an MIC. As shown earlier, the nonlocal TAT model only differs from the local TAT model at low electric fields (high voltage) and, thus, is useless for this test.

Fig. 3 shows that the technological layers and the real doping profile affect the response of the tunnel diode. Introduction of 


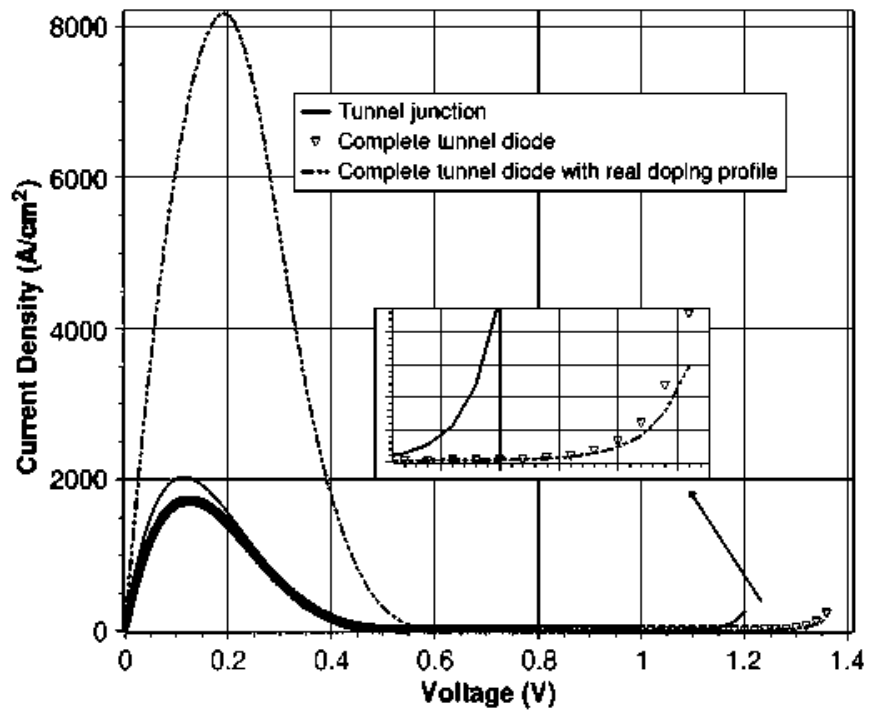

Fig. 3. Tunnel diode $J-V$ curves comparing the (solid line) aclive region simulation, (ttiangles) embedded diode, and (dashed line) embedded diode with a real secondary ion mass spectrometry profle.

these technological layers (i.e., the barrier layers, substrate, and cap layer) slightly decreases the peak current density from 2080 to $1720 \mathrm{~A} / \mathrm{cm}^{2}$, with a corresponding increase in the tunnel diode series resistance, from 0.06 to $0.07 \mathrm{~m} \Omega \cdot \mathrm{cm}^{2}$. The layers surrounding the tunnel diode impart recombination and current loss to the device. Moreover, it can be seen that these layers modify the threshold voltage of the diode from 1.15 to $1.3 \mathrm{~V}$. In this case, this modification is not relevant because the tunnel diode is intended to work in the first part of its $J-V$ curve, where it acts as a resistance. However, this change is important if we attempt to reproduce the behavior of this device in its entirety, rather than only where it is designed to work.

Comparison of the complete tunnel diode with and without the real doping profile demonstrates that the dopant profile strongly influences the characteristics of the device. The peak current density increases from 1720 to $8250 \mathrm{~A} / \mathrm{cm}^{2}$, and the peak voltage changes from 0.12 to $0.19 \mathrm{~V}$, with a corresponding decrease in the series resistance from 0.07 to $0.025 \mathrm{~m} \Omega \cdot \mathrm{cm}^{2}$. The threshold voltage remains intact and confirms that it has a stronger dependence on the layers around the tunnel junction than on the real doping profile of the tunnel junction. These simulations demonsirate the importance of the technological layers and the doping profile for reproducing the $J-V$ curves of the tunnel diode. As a result of these tests, we conclude than these two technological parameters of the device should be taken into account during the modeling of tunnel diodes.

\section{B. Effect of Trap Parameters}

The presence of trap levels inside the band gap affects the tunnel diode not only in the usual working region but also in the valley region. In this section, we will study the effects of the Iraps on the peak current in more detail and attempt to quantify them. The effect of the effective mass parameter $m_{\text {traps }}$ on the tunnel diode $J-V$ curve will be specifically examined.

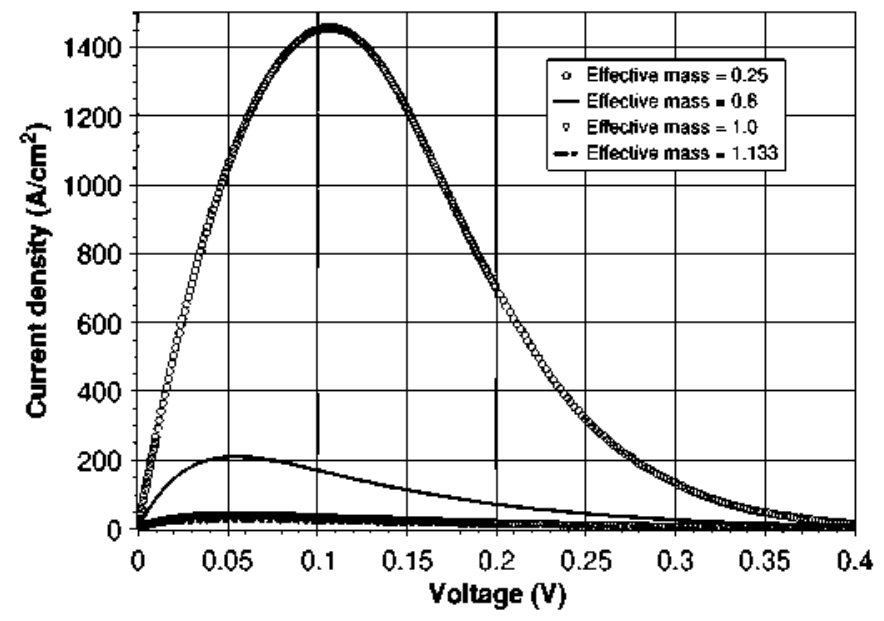

Fig. 4. Effect of the miraps parameter on the $J-V$ curve. The parameter is set to $0.25,0.6,1.0$. and 1.133 .

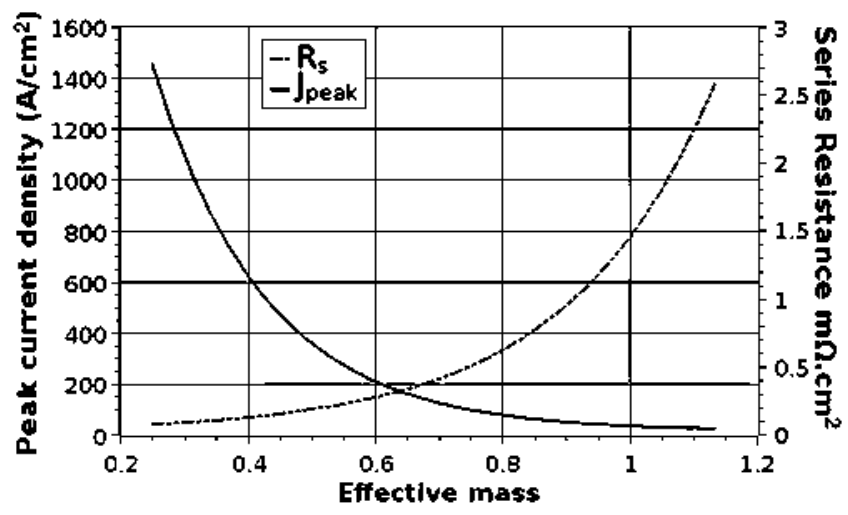

Fig. 5. Effect of the m.traps parameter on the peak curtent and on the series tesistance.

As we can see in (11) and (12), the field enhancement factor calculated for the local TAT model (and, thus, the peak current density) decreases with the square root of the effective mass. This parameter only affects the local TAT model because of the different approaches used to calculate $\Gamma_{n}^{\mathrm{DIRAC}}$ and $\Gamma_{p}^{\mathrm{DIRAC}}$ in the local and nonlocal TAT models. Nevertheless, these models yield equivalent peak current densities $J_{p}$.

In Fig. 4, the trap level is fixed to the midgap with a concentration of $1.5 \times 10^{14} \mathrm{~cm}^{-3}$. Neither the trap concentration nor the trap level is discussed in this section; they will be analyzed later when comparing the simulation data and experimental results. Fig. 4 summarizes the $J-V$ curves obtained when $m_{\text {traps }}$ is set to $0.25,0.6,1.0$, and 1.133. Fig. 5 shows the variation of both $J_{p}$ and the series resistance as a function of the effective mass in the trap level. $J_{p}$ is observed to exponentially decrease, whereas the series resistance exponentially grows with the effective mass. Moreover, it should be noted that the peak current is more strongly affected by changes in the effective mass than by changes in the series resistance. More precisely, the peak current is affected first by an increase in the effective mass, then it exponentially decreases to a saturated state; the series resistance then responds as the current (but not the peak voltage) continues to decrease, leading to a fast diminution of the series resistance. 


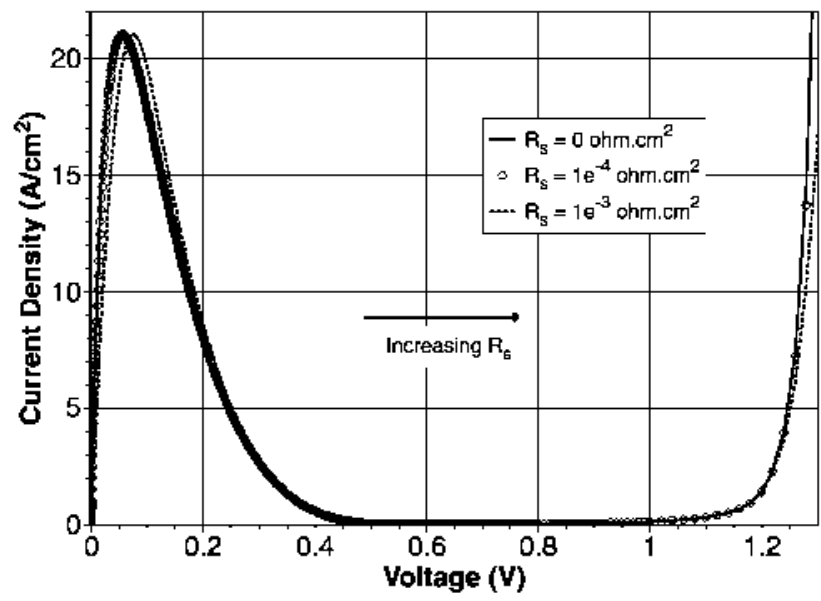

Fig. 6. Effect of the series resistance on the $J-V$ curve, $R_{s}$ was set to 0 . $1 \times 10^{-4}$, and $1 \times 10^{-3} \Omega \cdot \mathrm{cm}^{2}$.

\section{Series Resistance}

In this section, the effect of the series resistance on the $J-V$ curve is studied. The effects of the series resistance have becn previously studied [17] to show the effect of this component on the $J-V$ curve. The series resistance has been introduced into simulations to fit experimental data $[10]$ without determining the origin of the series resistance. A tunnel diode acts as a resistance near a zero bias voltage. This resistance can be regarded as two series-connected resistances: the tunnel diode component $R_{\mathrm{TD}}$, due to the tunnel junction, and the other scmiconductor layers; and a contact resistance $R_{c}$, duc to the metal, and the semiconductor/metal ohmic contact. Simulation of this effect on the tunnel diode characteristic should quantify the partitioning of the series resistance between the ohmic contacts and the inner tunnel diode. This partitioning must be accounted for when designing concentrator solar cells with high efficiencies at concentrations above 1000 suns.

Simulations were carried out for several values of $R_{c}$, and the results are shown in Fig. 6 . In this case, the $m_{\text {traps }}$ parameter has been set to 1.133 , leading to a global series resistance value of $2.6 \times 10^{4} \Omega \cdot \mathrm{cm}^{2}$ as it can been observed in Fig. 5. The choice for such a high value of the $m_{\text {traps }}$ parameter has been made to make the tunnel diode highly resistive and to see the sensitivity of the global resistance to small variation of $R_{e}$.

As expected, the value of $R_{c}$ does not affect the peak current density $J_{p} . R_{c}$ affects the slope of the voltage curve and translates the threshold voltage to higher values. An increase in $R_{c}$ results in a decrease in the slope of the curve for voltages above the threshold voltage. The inner resistance $R_{\mathrm{TD}}$ for this tunnel dioxle is $2.6 \times 10^{4} \Omega \cdot \mathrm{cm}^{2}$ in the voltage range $0-0.2 \mathrm{~V}$ $\left(J_{p}=210 \mathrm{~A} / \mathrm{cm}^{2}\right.$ at $\left.V_{p}=0.056 \mathrm{~V}\right)$. As we separate the tunnel diode into two series-connected resistances (an inner resistance $R_{\mathrm{TD}}$ and a contact resistance $R_{c}$ ), Ohm's law can bc applicd, giving

$$
V_{p}=R_{\mathrm{TD}} J_{p}+R_{c} J_{p}
$$

Because $J_{p}$ and $R_{\mathrm{TD}}$ are constant with respect to $V_{p}$

$$
\Delta V_{p}=\Delta R_{c} J_{p}
$$

TABLE I

TUNNEI. DIODE STRUCTURE

\begin{tabular}{llcl}
\hline Layer & Malerial & Thickness $(\mu m)$ & Doping $(c n-3)$ \\
\hline \hline CAP & GaAs & 0.5 & $\mathrm{p}-1 \mathrm{e} 18$ \\
Barrier & AlGaAs & 0.065 & $\mathrm{p}-2 \mathrm{e} 18$ \\
TD & GaAs & 0.020 & $\mathrm{p}-5 \times 10^{19}(\mathrm{C})$ \\
TD & GaAs & 0.020 & $\mathrm{n}-3 \times 10^{19}(\mathrm{Tc})$ \\
Barricr & AlGaAs & 0.09 & $\mathrm{n}-2 \mathrm{c} 18$ \\
Buffer & GaAs & 0.2 & $\mathrm{n}-2 \mathrm{c} 18$ \\
Substratc & GaAs & 300 & $\mathrm{n}-2 \mathrm{c} 18$ \\
\hline
\end{tabular}

where $\Delta V_{\text {peak }}$ is the peak voltage shift caused by the contact resistance $R_{c}$.

Determination of the series resistance components $R_{\mathrm{TD}}$ and $R_{c}$ is crucial. The inner tunnel junction series resistance $R_{\mathrm{TD}}$ will be transferred to the $\mathrm{MJC}$ or can be optimized by means of changes in the semiconductor structure. The contact resistance $R_{c}$ can be reduced by improving the metalization process.

\section{COMPARISON TO EXPERIMENTS}

The previous sections discussed the analysis of the effects of model and parameters on tunneling. It was shown that the doping profile and local TAT affect the peak current, the nonlocal TAT affects the valley region, and the series resistance due the specific contact resistance can alter the peak voltage. We now validate the model presented by comparison to experimental results.

\section{A. Manufacture of Tunnel Diodes}

We manufactured tunnel diodes, consisting of the tunnel junction, composed of two degenerately doped $\mathrm{n}^{++}$and $\mathrm{p}^{++}$ GaAs layers, and two enclosing barrier layers, with the purpose of minimizing dopant diffusion. The cap layer and the substrate were also included in the simulation. The metal-organic vaporphase epitaxy-grown tunnel diode used has the structure shown in Table I. Photolithography was used to fabricate the devices. The front contact is bascd on AuZn/Au, and the back contact is based on AuGc/Ni/Au. The cvaporated metal thickness is over $1 \mu \mathrm{m}$ to guarantec a homogencous current distribution on the front contact. The $J-V$ curves were measured using the fourpoint technique to avoid the introduction of the series resistance from the measurement tips.

\section{B. Results}

A proeedure was designed to fit the simulations to the experimental data. The aim of this method was 10 improve tunnel diode data fitling and to demonstrate the reliability of the models discussed here.

1) Include the complete layer structure of the tunnel diode in the simulation.

2) Include a realistic (or the real) doping profile.

3) Detect the presence (or absence) of traps from the experimental data, which can be done by focusing on the valley region of the tunnel diode characteristics. 


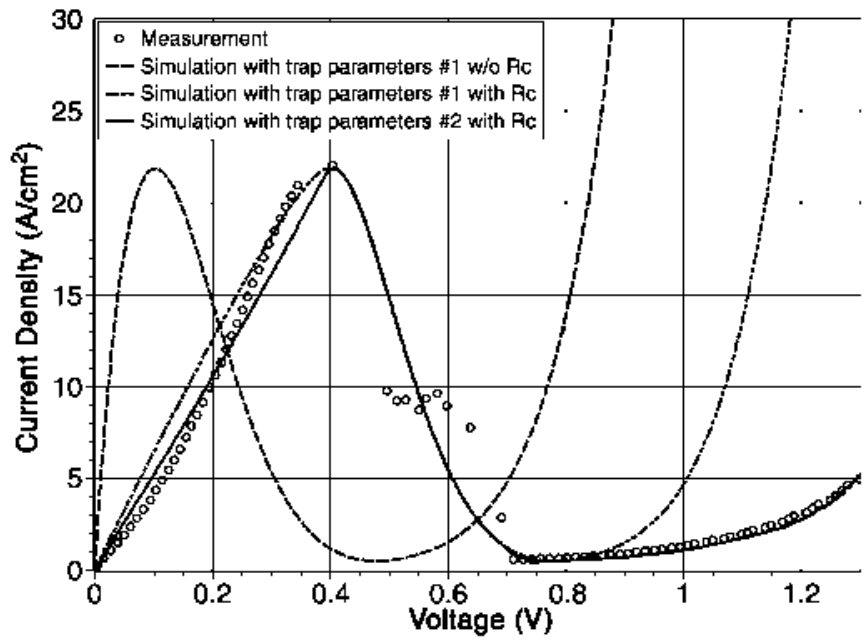

Fig. 7. Simulated and ineasured $J-V$ curves of the tunnel diode, including the series resistance $R_{c}$ and different trap parameters.

4) Simulate the slructure activating the nonlocal tunneling effects and nonlocal TAT with the default settings.

5) Enter a realistic trap concentration and effective mass, which allow matching of the peak current density $J_{p}$.

6) Vary the contact resistance $R_{c}$ to match $V_{p}$ ( $J_{p}$ has been matched in the previous step).

7) Readjust the trap level to match the valley region of the $J-V$ curve, adjusting the trap levels for the material used in the active region of the tunnel junction.

8) Readjust the trap concentration and effective mass to mateh the peak current density $J_{p}$.

We next present the results obtained from step 5 of the protocol as the first four steps were discussed in previous sections. Fig. 7 shows the experimental data and simulation results. The dashed lines represent the simulation that includes the first trap parameters both with and without series resistance. The effective masses (set to $m_{e}^{*}=0.159$ and $m_{h}^{*}=1.59$ ) and the values used for the trap paramelers are those of [20]. The results in [20] were obtained by simulating side-gating effects in GaAs MESFETs with one donor state and gave exeellent values for the following parameters:

$$
E_{T}=0.715 \mathrm{eV} \text { and density of traps }=1.5 \times 10^{14} \mathrm{~cm}^{3}
$$

where $E_{T}$ is the separation between the trap level and the conduction band. We can see that the peak current density is perfectly matched with these trap parameters. It can also be seen that the addition of a series resistance allows a good match between the model and experimental data for voltages up to $0.8 \mathrm{~V}$. In this case, a contact resistance of $R_{c}=0.0135 \Omega$. $\mathrm{cm}^{2}$ has been added. However, the valley regions of the $J-V$ curves strongly differ between the experimental data and the simulation results, probably due to the trap parameters.

Although these parameters reproduce the tunnel diode characteristic until $J_{p}$, they are not very accurate for voltages above $V_{p}$. For MJCs, these results are not sufficiently precise. Under normal operation conditions, the tunnel junction should act as a resistance, and this behavior is reproduced here. However, it is crucial to properly simulate the $J-V$ curve to predict the performance of solar cells inside optical concentrators.

According to (15), a contact resistance $R_{c}=0.0135 \Omega \cdot \mathrm{cm}^{2}$ can he calculated and included in the simulation. Concerning the trap parameters, from the extensive study of trap levels in [21], a deep defeet level corresponding to carbon-doped GaAs was found to produce a better fit with experimental data (when considering the same effective masses as before), i.c.,

$$
E_{T}=0.3 \mathrm{cV} \text { and density of traps }=2.75 \times 10^{14} \mathrm{~cm}^{-3} \text {. }
$$

Fig. 7 shows the results obtained by changing the trap parameters. As can he seen, the addition of the contact resistance $R_{c}$ allows a perfect fit of the peak voltage $V_{p}$. Fig. 7 also shows a difference produced hy the change in the traps properties. These parameters affeet not only the tunneling effect but also the response of the tunnel diode at low electric fields. Accurate matcrial parameters, reliable information aboul the doping concentrations, and the type of impurities present are important for the behavior. It should be noted that this fitting procedure is not only compatible mathematically but also technologically. Carbon is the dopant used in the p-side tunnel junction, creating a trap level inside the band gap of GaAs. This observation demonstrates the suitability of the model. Introduction of these trap parameters leads to a perfect fit between simulated and experimental data,

Fig. 7 also highlights the small differences between simulation and experiment in the negative resistance region for voltages between 0.4 and $0.7 \mathrm{~V}$. As shown in [17], this region of the tunnel diode $J-V$ curve is difficult to measure, and the deviations probably result from measurement artifacts.

The voltage drop caused by the tunnel junction in an MJC is the value of the voltage corresponding to the maximum current density that the solar cell can give once read onto the tunnel diode $J-V$ characteristic. The predictive utility of the simulations is apparent from the tunnel diode, which shows a very high contact resistance $R_{c}$. A maximum shorleireuil current densily $J_{\mathrm{sc}}$ of $13 \mathrm{~mA} / \mathrm{cm}^{2}$ (a typical value for GaInP/GaAs dual-junction solar cells at 1000 suns) would result in an experimentally observed voltage drop of $0.24 \mathrm{~V}$. This voltage drop is very high and would have negative effects on the operation conditions of the solar cell. However, the simulation results suggest that the largest voltage drop is caused by the contact resistance. Fig. 7 reveals that the experimental voltage drop caused by the tunnel diode is $0.04 \mathrm{~V}$, which is a more reasonable value. Previous related works [10] have modeled tunnel diodes with very good results. These works show an exeellent fit around the prak current density and in the thermal current region of the diode. However, the fit was not as good in the valley region of the $J-V$ curve, in contrast to the model presented here. This model demonstrates an excellent fit throughout the whole voltage range. Finally, it should be noted that the model presented mainly depends on the band diagram and on the trap parameters. In theory, this model should be applicable to all types of semiconductor materials for which good parameters and trap information are available. 


\section{SUMMARY AND CONCLUSION}

In this paper, we have reviewed the mechanisms involved in the tunneling effect. We have detailed the infuence of the local and nonlocal TAT effects on the tunnel diode characteristics. We have described the effects of Irap concentration and effective masses on the peak current density and the degree to which the trap level modifies the low electric field response of the diode characteristic (the valley region). We have developed a protocol for fitting the measured $J-V$ curves and demonstrated the agreement between measurement and simulation. We have explained a method for extracting the voltage drop that the MJC will experience due to the tunnel diode by separating the tunnel diode resistance into two components and by allowing the components to be calculated from the tunnel diode itself and from the metalization process. Finally, his paper shows a reasonable fit between experiment and simulation data over the whole voltage range, which is particularly important for the case of MJCs. A nonuniform light distribution will cause the tunnel junctions inside solar cells to work at different regimes, necessitating a model that can perfectly reproduce the entire tunnel diode $J-V$ curve.

\section{ACKNOWLEDGMENT}

The authors would like to thank the Silvaco Grenoble Research Ceuter (G.RE.CE.) for its collaboratiou. The authors would also like to thank I. Rey-Stolle, B. Galiana, I. García, aud P. Espinet of the IES-UPM, who made the experimental data available. The Spanish Ministerio de Ciencia e Innovacion has also contributed with the SIGMASOLES project (PSS-4400002009-30) and with the project with reference TEC2008-01226, as well as the Comunidad de Madrid under the NUMANCIA II programme (S2009/ENE1477). The European Union has also coutributed by means of the Europeau Fuud for Regional Developineut (FEDER).

\section{REFERENCES}

[1] J. P. Sun, G. I. Haddad, P. Mazumber. and J. N. Schulnan, "Resonant tunneling diodes: Models and properties," Proc. IEEE, vol. 86, no. 4, pp. $641-660$. Apr. 1998.

[2] W. Guter. J. Schöne, S. P. Philipps. M. Stejner. G. Siefer. A. Wekkeli. E. Welser, E. Oliva, A. W. Bett, and F. Dimroth, "Current-matched triplejunction solar cell reaching $41.1 \%$ conversion efficiency under concenirated sunlight." Appl. Phys. Lett. vol. 94. no. 22, p. 223504 . Jun. 2009. DOI: $10.1063 / 1.3148341$.

[3] C. Alyota, I. Rey-Stolle, I. García, B. Galiana, M. Baudrit, P. Espinet, E. Baurigón. A. Dauas, J. R. Gonzälez, and J. Bautista. "A dual junction solat cell with an efficiency of $32.6 \%$ at $1000 \mathrm{X}$ and $31.0 \%$ at $3000 \mathrm{X}$." in Proc. 5th ICSC. Palm Deser, CA, 2008, to be published.

[4] M. Baudrit and C. Algora. "3D modeling of concentrator III-V multijunction solat cells," in Pro. Rec. IEEE 4th WCPEC, 2006, vol. 1/2, Pp. 826-829.

[5] M. Baudrit, C. Algora, I. Rey-Stolle, I. Garcia, and B. Galiana, "Numerical analysis of GaInP solar cells: Toward advanced photovoltaic devices modeling," in Proc. 5th NUSOD, Berlin, Germany, 2005, pp. 41-42.

[6] G. Letay. M. Hertule, and A. W. Bett. "Simulating single-juncion research GaAs solat cells including photon tecycling." Prog. Photovolt.: Res. Appl, vol. 14, no. 8. pp. 683-696, Dec. 2006.

[7] G. A. M. Hurkx. D. B. M. Klaassen, and M. P. G. Knuvers, "A new reconbinaijon inodel for device sinulation including tunneling," IEEE Trans. Electron Devices, vol. 39, no. 2. pp. 331-338, Feb. 1992.

[8] E. O. Kane, "Theory of tunneling." J. Appl. Phys., vol. 32, no. 1, pp. 8391 , Jan. 1961
[9] R. Gelinas, "A novel approach to modeling tunnel junction diodes using Silvaco ATLAS software." M.S. thesis, Naval Postgraduate School. Monterey, CA, 2005.

[10] M. Herinle, G. Letay. S. P. Philipps, and A. W. Bett, "Numerical simulalion of tunnel diodes for inulti-junction solar cells," Prog. Photovolt: Res. Appl., vol. 16, no. 5, pp. 409-418, Aug. 2008.

[11] ATLAS User"s Manual. Silvaco. Sania Clara. CA, Sep. 2008.

[12] M. Baudrit and C. Algora, "Theoretical optimization of $\mathrm{GaInP} / \mathrm{GaAs}$ dual-junction solar cell: Toward a $36 \%$ efficiency at 1000 suns," Phys. Stat. Sol. (A), vol. 207, no. 2, Pp. 474-478, Feb. 2010. DOI: $10.1002 / \mathrm{pssa} .200925210$.

[13] L. Esaki, "New phenomenon in nartow Getmanium p-n junction," Phys. Rev., vol. 109, no. 2, pp. 603-604, Jan. 1958.

[14] G. Wentzel. "Eine Verallgeneinerun der Quantenbedingungen für die Zwecke det Wellenmechanik," Zeits. f. Phys., vol. 38, pp. 518-529, 1926.

[15] H. A. Kramers, "Wellenmechanik und halbzahlige Quantisierung." Zeits. f. Phys. vol. 39, pp. 828-840, 1926 .

[16] L. Brillouin, "La mécanique ondulatoite de Schrödinger; une méthode générale de resolution par approximations successives," Comptes Rendus, vol. 183, no. 1, pp. 24-26, 1926 .

[17] W. Guter and A. W. Bett, "I-V characterization of tunnel diodes and multijunction solar cells," IEEE Trans. Electron Devices, vol. 53, no. 9 , pp. $2216-2222$, Sер. 2006

[18] T. Takalnoto, M. Yulnaguchi. E. Ikeda, T. Aguj, H. Kurita, and M. Al Jassim, "Mechanism of $\mathrm{Zn}$ and Si diffusion from a highly doped tunnel junction for InGaP/GaAs tandem solar cells." J. Appl. Phys. vol. 85. no. 3, pp. 1481-1486, Feb. 1999.

[19] N. Kojima, M. Okamoto, S. J. Taylor, M. J. Yang, T. Takamoto, M. Yamaguchi, K. Takahashi, and T. Unno. "Analysis of inpurity diffusion from tunnel diodes and optinization for operation in tandem cells." Sol. Energy Mater. Sol. Cells, vol. 50, no. 1-4, pp. 237-242, Jan. 1998.

[20] N. Goto. Y. Obno, and H. Yano, "Two-dimensional numerical simulation of side-gating effect in GaAs MESFETs," IEEE Traus. Electron Devices, vol. 37, no. 8. pp. 1821-1827, Aug. 1990.

[21] J. W. Huang, K. L. Bray, and T. F. Kuechb. "Compensation of shallow inpurities in oxygen-doped metalorganic vapor phase epitaxy grown GaAs." J. Appl. Phys., vol. 80, no. 12. pp. 6819-6826. Dec. 1996

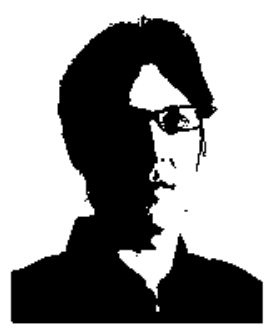

Mathien Bandrit received the M.S. degree from the National Institute for Applied Sciences of Lyon (INSA). Lyon. France, in 2003 and the Ph.D. degree from the Universidad Politécnica de Madrid. Madrid, Spain.

Since October 2010 , he has been with the French National Institute for Solar Energy (INES), Le bourget du Lac, France, involved in beterojunction (amorphous silicon on silicon). homojunction (silicon) and quantum-dot solar cell simulation. His tesearch interespecially silicon based solar cells. ests are parlicularly focused on solar cell modeling.

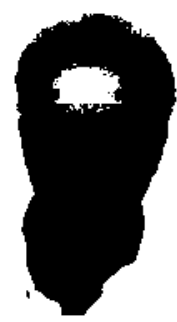

Cartos Algora (SM'98) recejved the B.Sc. and Ph.D. degrees in physics from the Universidad Complutense de Madrid, Madrid, Spain, in 1986 and 1990 , respectively.

In 1985, he joined the Departamento de Electrónica Física, Instituto de Energía Solar, Universidad Politécnica de Madrid (UPM), Madrid, where he has been a Full Professor since 2008. Since 1996, has been the Head of the Group III-V Semiconductors. $\mathrm{He}$ has contributed to several European and world efficiency records on III-V concentralor solar cells. He has been the main researcher of more than $40 \mathrm{R} \& \mathrm{D}$ projects. He has published 200 scientific papers. He is the holder tive patents.

Prof. Algora was the recipient of the 2009 Research Prize from UPM. 